\title{
Многолетние колебания температуры поверхности Мирового океана в связи с изменениями геомагнитной активности
}

\author{
Б. Г. Шерстюков ${ }^{1 \bowtie}$ Ю. П. Переведенцев²
}

${ }^{1}$ Всероссийский научно-исследовательский институт гидрометеорологической информации Мировой иентр данных, Российская Федераџия (249035, г. Обнинск, Калужская обл., ул. Королева, 6)

${ }^{2}$ Казанский Федеральный университет, Институт экологии и природопользования, Российская Федераџия (420097, г. Казань, ул. Товарищеская, 5)

Поступила в редакиию 31.01.2020.

Принята к публикации 26.02.2020

\begin{abstract}
Аннотация: Цель. Предполагалось получить оценки связи колебаний температуры поверхности Мирового океана с изменениями геомагнитной активности с учетом пространственно-временных особенностей.

Memoдbl. Применялся метод асинхронного корреляционного анализа связи температуры океана в узлах географической сетки с изменениями геомагнитной активности за 1868-2018 годы.

Pезультаты. Положительные корреляции температуры с геомагнитной активностью обнаружены на Антарктическом течении в районе пересечения им Восточно-Тихоокеанского поднятия дна океана (запаздывание 32-33 года), а также на пересечении Антарктическим течением южного окончания Срединно-Атлантического хребта (запаздывание 22-26 лет) и в узком месте между Австралией и Антарктидой (запаздывание около 30 лет). Такие же высокие корреляции получены в центральной экваториальной части Тихого океана, в том районе, в котором появляются Эль-Нинье и Ла-Нинье (запаздывание около 43 лет), и в районе Южного колебания (запаздывание 42-44 лет). Отрицательные корреляции обнаружены в Мексиканском заливе (запаздывание 35-36 лет), в районе пересечения Северо-Атлантическим течением Срединно-Атлантического хребта (запаздывание 32-34 года) и в районе пересечения Бенгельским течением Китового подводного хребта (запаздывание 37-39 лет). В Тихом океане отрицательные корреляции обнаружились в районе пересечения Северо-Тихоокеанским течением СевероЗападного подводного хребта (запаздывание 39-44 года) и вблизи прохождения Восточно-Австралийским течением островов Полинезии (запаздывание 37-39 лет).

Bblводы. В районах основных океанических течений обнаружены тесные связи изменения температуры поверхности океана с возмущениями магнитного поля Земли. Предполагается воздействие на течения электрических токов, которые возникают в океане при магнитных бурях по аналогии с известными явлениями в магнитосфере и ионосфере. Запаздывание изменений температуры на поверхности объясняется временем передачи возмущений с больших глубин океана на поверхность.
\end{abstract}

Ключевые слова: солнечная активность, геомагнитная активность, температура океана, колебания климата, корреляционный анализ.

Источник финансирования: Работа выполнена при финансовой поддержке Российского фонда фундаментальных исследований, проекты 18-05-00721 и 18-45-160006.

(C) Шерстюков Б.Г., Переведенцев Ю. П., 2020

E-mail: boris@meteo.ru

Контент доступен под лицензией Creative Commons Attribution 4.0 License.

The content is available under Creative Commons Attribution 4.0 License. 


\title{
Long-Term Fluctuations in the Surface Temperature of the World Ocean due to Changes in Geomagnetic Activity
}

\section{B. G. Sherstyukov ${ }^{1 凶}$, Yu. P. Perevedentsev ${ }^{2}$}

\begin{abstract}
Purpose. It was supposed to obtain estimates of the relationship between temperature fluctuations on the surface of the oceans and changes in geomagnetic activity, taking into account spatial and temporal features.

Method. The method of asynchronous correlation analysis of the relations between the sea surface temperature at the geographic grid and changes of geomagnetic activity for 1868-2018 was used.

Results. Positive correlations of temperature with geomagnetic activity were found on the Antarctic current in the region were it intersects of the East Pacific Rise of the ocean floor (with delay 32-33 years), as well as on the intersection by Antarctic currents of the southern end of the Mid-Atlantic Ridge (with delay 2226 years) and in the narrow of ocean between Australia and Antarctica (about 30 years late). The same high correlations were obtained in the central equatorial part of the Pacific Ocean, in the region where El Nine and La Nine appear (a delay of about 43 years), and in the region of the Southern Oscillation (a delay of 4244 ears). Negative correlations were found in the Gulf of Mexico (with 35-36 year delay) in the region were North Atlantic current crosses the Mid-Atlantic Ridge (32-34 year delay). The same correlation were on the Bengal current crosses Whale Range (37-39 year delay). In the Pacific Ocean, negative correlations were found at the intersection of North-West underwater ridge by the North-Pacific current (the delay of 3944 years) and negative correlations on the East Australian current near of the islands of Polynesia (delay of 3739 years).

Conclusions. In the areas of the main ocean currents, close connections were found between changes in the surface temperature of the ocean and disturbances in the Earth's magnetic field. The effect on the ocean currents of electricity that occur in the ocean during magnetic storms is assumed, by analogy with the known phenomena in the magnetosphere and ionosphere. The delay in surface temperature changes is explained by the time of disturbance transmission from large depths of the ocean to the surface.
\end{abstract}

Key words: solar activity, geomagnetic activity, ocean temperature, climate fluctuations, correlation analysis.

Funding: The work was supported by the Russian Foundation for Basic Research, projects 18-05-00721 and 18-45-160006.

\section{ВВЕДЕНИЕ}

Колебания являются фундаментальным свойством климата и наблюдались во все времена, однако причины появления этих колебаний и факторы их поддерживающие, остаются недостаточно изученными. Таких причин предполагается несколько. Одним из наиболее давних предположений является гипотеза о влиянии солнечной активности на состояние климата. Под солнечной активностью понимается комплекс явлений, происходящих в недрах Солнца и на его поверхности, а также модулированные Солнцем явления в межпланетном пространстве, в магнитосфере и в других оболочках Земли. Нельзя изучать влияние солнечной активности на климат, основываясь только на сведениях об изменении светимости Солнца.

Геомагнитные возмущения, регистрируемые на Земле, являются индикатором активности Солнца и его влияния на Землю при взаимодействии с магнитосферой Земли заряженных частиц, приходящих из активных областей Солнца. Траектории потоков заряженных частиц не всегда пересекаются с Землей и поэтому не каждый поток частиц геоэффективен. Появление геомагнитных возмущений - это гарантия того, что энергия солнечной активности достигла Земли.

Сложный комплекс солнечной активности соприкасается с не менее сложной климатической системой, в которой характеристики климата атмосферы являются следствием неизвестной последовательности неизученных процессов превращения изменяющейся активности Солнца в изменения температуры воздуха и других характеристик. Рассматривая составляющие климатической системы и их взаимодействия, более уверенно можно говорить о том, что Мировой океан является генератором изменений климата атмосферы, но не наоборот. В работе [6] описаны долгопериодные колебания температуры воздуха в республике Татарстан, которые следуют за колебаниями температуры поверхности Мирового океана с запаздыванием. На большом фактическом материале показана статистическая достоверность асинхронных коэффициентов корреляций. По данным [8] за 1961-2003 годы произошло увеличение теплосодержания климатической системы на $15,9 * 10^{22}$ 
джоулей. Из них увеличение теплосодержания океана произошло на $14,2 * 10^{22}$ джоулей, атмосферы - на $0,5 * 10^{22}$ джоулей, суши - на 0,76*1022 джоулей, льда - на $0,45 * 10^{22}$ джоулей. Из общего увеличения теплосодержания 89,3\% пришлось на океан и только 3,1\% на атмосферу.

На основе приведенных сведений показатель геомагнитной активности выбран в качестве характеристики влияния солнечной активности на Землю, а на Земле Мировой океан выбран в качестве места наиболее вероятного проявления изменений геомагнитной активности.

\section{МАТЕРИАЛЫ И МЕТОДЫ ИССЛЕДОВАНИЯ}

В работе использованы среднегодовые данные планетарного геомагнитного индекса аа за 18682018 годы [10] и среднегодовые данные о температуре поверхности Мирового океана (ТПО) в узлах географической сетки через два градуса по широте и долготе за 1901-2018 годы, массив ERSST v3b. Массив данных ERSST v3b представляет собой восстановленные ряды месячных аномалий ТПО относительно норм за 1971-2000 годы [12].

Наиболее яркой особенностью многолетних изменений геомагнитной активности является 11-летний цикл. В работе основное внимание уделено поиску проявления этой 11-летней цикличности в изменениях ТПО, поэтому методом скользящих средних по пятилетиям были сглажены колебания индекса $а a$ с периодами менее 5 лет. А удаление линейного тренда из $a a$ за 1868-2008 годы позволило избавиться от сверх вековой тенденции. Ряд ТПО был обработан скользящими средними аналогично, а линейный тренд исключен за 19012018 годы. Далее эти обработанные ряды будем также называть $a а$ и ТПО, помня, что в них сглажены короткопериодные вариации и исключены сверх вековые тренды.

Применялся метод асинхронного корреляционного анализа ряда индекса $а a$ с рядами ТПО в каждом узле сетки Мирового океана. Многолетние изменения ТПО определяются глубинными процессами в океане, которые обладают многолетней инерционностью, поэтому поиск проявления геомагнитных колебаний в ТПО проводился в предположении возможного запаздывания изменений ТПО относительно изменений $а a$. Запаздывание допускалось до 50 лет.

Допущение такого запаздывания основано на нескольких публикациях. Существует глобальная система океанической циркуляции (Большой конвейер), которая перераспределяет тепло по всему миру. Теория Большого океанического конвейера утверждает, что региональные океанические течения объединены в одну общую систему [7]. Двигателем конвейера является термохалинная циркуляция. Большой конвейер океанических течений медленно переносит тепло и влагу из одних регионов и слоев океана в другие по сложным, не совсем понятным траекториям. Выход аномалий температуры на поверхность океана в разных его частях возможен с запаздыванием в несколько лет или десятилетий. Допускается существование колебаний климата, связанных с ослаблением и усилением термохалинной циркуляции с периодами от нескольких лет до порядка 1000 лет [2, 3, 7]. Термохалинная циркуляция является глобальным явлением, распространяющимся на Атлантический, Индийский и Тихий океаны. Анализируя асинхронную ковариационную структуру климатических индексов, в работе [13] описывается распространение сигнала изменения температуры по всему Северному полушарию через последовательность многократных превращений асинхронных атмосферно-океанических дальних связей. По оценкам тех же авторов, первоначальный сигнал изменения температуры в Северной Атлантике, распространяясь, достигает удаленных регионов полушария примерно 30 лет спустя.

В работе [1] на основе других подходов показано, что отклик состояния атмосферы на внешнее воздействие происходит с запаздыванием, которое определяется необходимым временем релаксации, свойственным всей климатической системе. Оптимальные значения такого времени релаксации в [1] получены 25,1-27,5 лет. Близкие оценки запаздывания обнаружены и при других циклических внешних воздействиях на океан. Так, например, в [14] обнаружено влияние циклических изменений солнечной активности на СевероАтлантическое колебание с запаздыванием в 40 лет в результате смещения зоны тропической конвекции в Тихом океане.

При вычислениях асинхронных корреляций текущее состояние ТПО сравнивалось со значениями $a a$, которые наблюдались ранее. Число значений каждого ряда, по которым вычислялась корреляция, было 118 при нулевом сдвиге и на 12 значений меньше при максимальном сдвиге. При всех сдвигах до 33 лет потери сопоставляемых значений не происходило и их количество оставалось всегда 118, так как ряд индекса аa начинается на 33 года раньше ряда ТПО. При таком количестве значений рядов, с учетом связанности сглаженных 


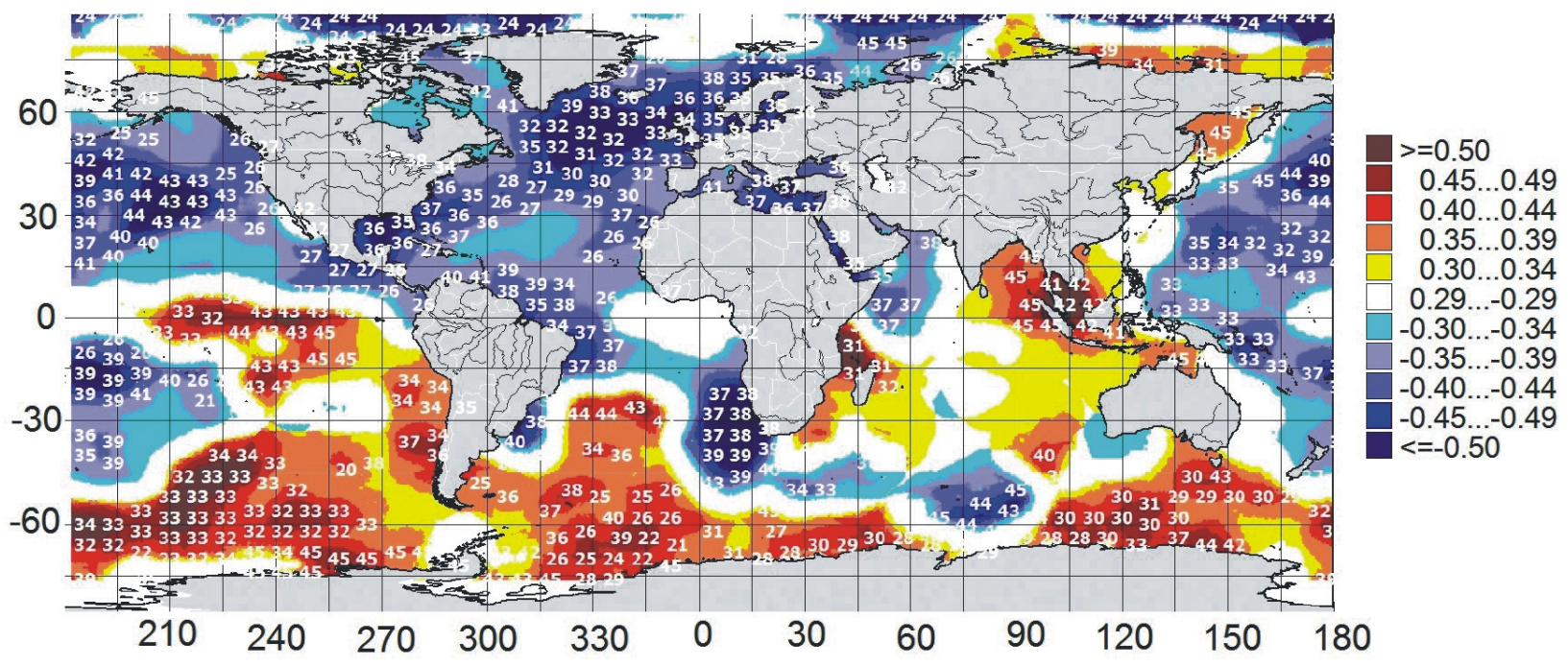

Puc. 1. Поля коэффициентов наилучшей асинхронной корреляции ТПО с индексом геомагнитной активности аа и значения запаздывания (количество лет запаздывания показано цифрами) изменений ТПО относительно изменений $a a$

[Fig. 1. Fields of coefficients of the best asynchronous correlation of the surface temperature of the World ocean with the geomagnetic activity index $a a$ and the lag value (the number of years of lag is shown in figures) of changes in the surface temperature of the World ocean relative to changes in $a a$ ]

значений, статистически достоверными являются коэффициенты корреляции 0,4 и выше с вероятностью $95 \%$.

Корреляции вычислялись при сдвигах ряда индекса аа от 0 до 50 лет. При этом в каждом узле из каждой серии асинхронных корреляций находился максимальный по модулю коэффициент и фиксировался сдвиг, при котором этот коэффициент был получен. Затем строилась карта полей вычисленных наилучших корреляций с нанесением на нее цифрами величин сдвигов в каждом узле со значимым значением коэффициента наилучшей асинхронной корреляции.

\section{ОБСУЖДЕНИЕ РЕЗУЛЬТАТОВ}

Карта наилучших асинхронных корреляций аа и ТПО показала, что максимальные по модулю значимые корреляции обнаруживаются только при сдвигах от 20 до 44 лет (рис. 1).

Bce обширные очаги наилучших корреляций оказались в районах вдоль траекторий основных океанических течений. В Северном полушарии преобладают отрицательные корреляции на теплых течениях: в Атлантическом океане - Гольфстрим, Северо-Атлантическое течение, Норвежское течение; в Тихом океане - Куросио, Северо-Тихоокеанское течение, а в Южном полушарии значимые положительные корреляции обнаруживаются на холодных течениях, главным и самым мощным из которых является циркумполярное Антарктическое течение (Западных ветров течение), проходящее по южным районам Тихого, Атлантического и Индийского океанов.

Примечательно, что самые высокие коэффициенты корреляции, по модулю превышающие 0,50,6 и до 0,7 обнаруживаются в тех местах океанов, в которых основные течения пересекают подводные хребты или проходят через гряды островов. Так, например, темными пятнами высоких отрицательных корреляций на карте (рис. 1) в Атлантическом океане отметились: узкое место выхода Гольфстрима из Мексиканского залива (запаздывание 35-36 лет), район пересечения СевероАтлантическим течением Срединно-Атлантического хребта в Северной Атлантике (запаздывание $32-$ 34 года) и район пересечения Бенгельским течением Китового подводного хребта в Атлантическом океане юго-западнее Африки (запаздывание 37-39 лет). В Тихом океане самые высокие отрицательные корреляции обнаружились в районе пересечения Северо-Тихоокеанским течением Северо-Западного подводного хребта и далее по течению с переходом на прилегающий район северной части Северо-Восточной котловины (запаздывание 39-44 года). На юге Тихого океана высокие коэффициенты корреляции видны на карте вблизи прохождения Восточно-Австралийским течением островов Полинезии (запаздывание 37-39 лет).

Высокие положительные корреляции 0,5-0,6 и выше обнаружились на Антарктическом течении в районе пересечения им Восточно-Тихоокеанско- 


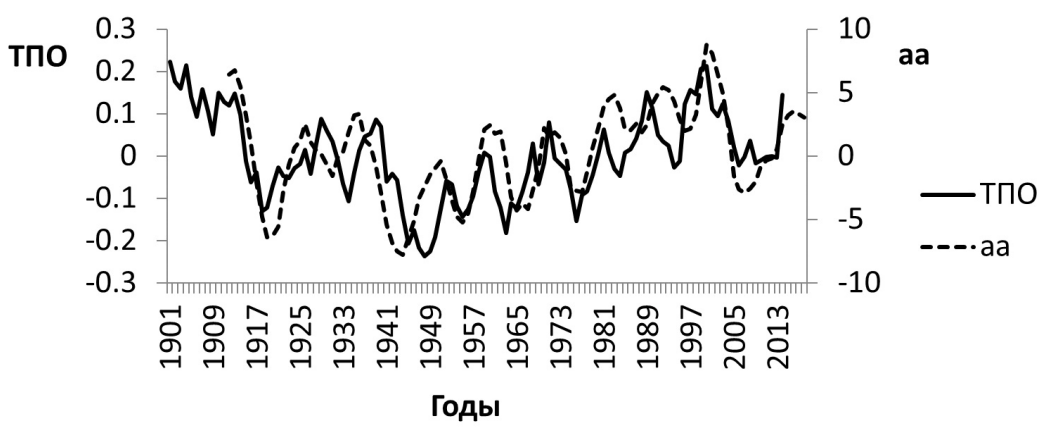

Puc. 2. Изменение ТПО в географическом узле с координатами $4^{\circ}$ с.ш. и $104^{\circ}$ в.д. и изменение планетарного геомагнитного индекса аa со сдвигом на 42 года

[Fig. 2. Changes in the surface temperature of the World Ocean at the geographical node with coordinates $4^{\circ} \mathrm{n} .1$. and $104^{\circ}$ e.l. and changes in the planetary geomagnetic index $a a$ with a shift of 42 years]

го поднятия дна океана (запаздывание 32-33 года), а также на пересечении Антарктическим течением южной оконечности Срединно-Атлантического хребта (запаздывание 22-26 лет) и в узком месте между Австралией и Антарктидой (запаздывание около 30 лет). Такие же высокие корреляции видны на карте в центральной экваториальной части Тихого океана, в том районе, в котором появляются Эль-Нинье и Ла-Нинье (запаздывание около 43 лет), и в районе активного взаимодействия Индийского и Тихого океана, с известным Южным колебанием (запаздывание 42-44 лет).

Во всех перечисленных случаях наилучшие асинхронные корреляции наблюдались при запаздывании изменений ТПО относительно аа на 2 или 3 или 4 одиннадцатилетних цикла. При наличии известной 11-летней цикличности можно заподозрить ошибочный статистический эффект сдвига на один или несколько циклов, но индекс аа кроме 11-летнего цикла еще содержит вековой цикл, который не дает ошибочно сместиться на 1-3 одиннадцатилетних цикла. Подробнее остановимся на двух примерах.

На рисунке 2 показан график временного хода ТПО в районе Южного колебания (точка с координатами $4^{\circ}$ с.ш. и $104^{\circ}$ в.д.) и график изменения aа, сдвинутый на 42 года в будущее. Это пример положительной связи. Коэффициент корреляции $\mathrm{R}=0,66$. А на рисунке 3 показан график ТПО в Атлантике юго-западнее Африки (точка с координатами $28^{\circ}$ ю.ш. и $10^{\circ}$ в.д.) и график изменения аa, сдвинутый на 38 лет в будущее. Это пример отрицательной связи. Коэффициент корреляции $\mathrm{R}=-0,70$.

Высокие корреляции являются доказательством неслучайной связи ТПО и $а a$. Рисунки 2 и 3 показывают, что при выделенных наилучших сдви- гах совпадают циклические изменения ТПО и $a a$, как в одиннадцатилетнем цикле, так и в вековом цикле. Совпадение в вековом цикле не позволяет ошибочно сдвинуться на один или более одиннадцатилетних циклов. За рассмотренный интервал 118 лет наблюдалось 10 одиннадцатилетних циклов активности аа (рис. 2, 3) и 10 видимых на этих рисунках откликов в изменениях ТПО. Совпадение десять из десяти не может быть случайным. Обнаруженные зависимости и описанное выше длительное запаздывание реакции ТПО являются реальностью.

Обнаруженное запаздывание реакции поверхностной температуры на глубинные изменения в океане при геомагнитных возмущениях согласуется с известными свойствами океана. В разных частях Мирового океана различия во времени реакции поверхностной температуры на геомагнитные возмущения могут быть связаны с региональными условиями динамических процессов во всей толще океана и с рельефом дна, а также с конфигурацией континентов.

Реальность обнаруженных связей подтверждается не только статистически по высоким значимым коэффициентам корреляции, но и физическими особенностями проявления обнаруженных связей. Карта рисунок 1 уверенно демонстрирует, что все самые тесные зависимости ТПО от $а a$ проявляются на основных океанических течениях, а не разбросаны хаотично по Мировому океану.

Возникает вопрос о механизме передачи энергии, связанной с изменениями геомагнитной активности, в изменения ТПО Мирового океана на течениях.

Предположения о механизме можно строить исходя из основных свойств явлений в токопроводящих средах Земли, возникающих при геомагнит- 


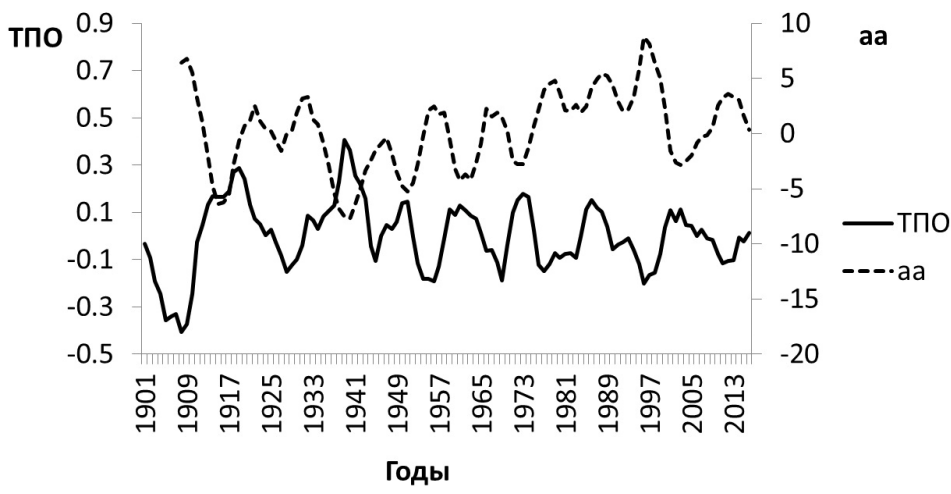

Puc. 3. Изменение ТПО в географическом узле с координатами $28^{\circ}$ ю.ш. и $10^{\circ}$ в.д. и изменение планетарного геомагнитного индекса $a а$ со сдвигом на 38 лет

[Fig. 3. Changes in the surface temperature of the World ocean at the geographical node with coordinates $28^{\circ} \mathrm{s} .1$. and $10^{\circ}$ e.l. and changes in the planetary geomagnetic index $a a$ with a shift of 38 years]

ных возмущениях планетарного масштаба. Геомагнитные возмущения являются следствием высыпания заряженных солнечных частиц в магнитосферу Земли и появления магнитных бурь. В моменты магнитных бурь эти частицы взаимодействуют с магнитосферой-ионосферой Земли и создают ионосферные токи в миллионы ампер по величине, а также вызывают индуцированные токи в проводящих средах на Земле и даже в проводящих технологических конструкциях (энергетические системы, трубопроводы, кабельные сети) [5]. Зафиксировано, что в технологических конструкциях во время магнитных бурь наблюдались геоиндуцированные токи до 200-300 А [11]. Хотя наиболее мощные возмущения геомагнитного поля, приводящие к возбуждению интенсивных токов, происходят в высоких широтах, в последнее время обнаружено, что опасные величины индуцированных токов могут наблюдаться также на средних и низких широтах [9].

Новые исследования [4] подтвердили наличие геомагнитно-индуцированных токов в проводящих средах на Земле не только в широтном, но и в долготном направлениях.

Океан является проводящей средой и в нем неизбежно должны появляться индуцированные токи преимущественно в широтном направлении в высоких широтах Северного и Южного полушарий, а также в других направлениях. Судя по большому запаздыванию изменений ТПО после усиления геомагнитной активности в 11-летнем цикле, в передаточном механизме участвуют, прежде всего, глубинные слои Мирового океана. А передача этих возмущений па поверхность происходит с многолетним запаздыванием, которое соответствует скоростям распространения возмущений в океане, приведенным выше в разделе выбора метода исследования. Об участии глубинных слоев океана в этом механизме говорит и тот факт, что самые тесные связи температуры на поверхности с геомагнитными возмущениями обнаружены в местах пересечения течениями подводных хребтов. В этих местах подводные препятствия на пути океанических течений усиливают вертикальный обмен в океане и усиливают вынос глубинных возмущений на поверхность океана. Следовательно возмущения ТПО действительно идут из глубин океана, а на их вертикальное распространение требуется время. И, следовательно, обнаруженное запаздывание реакции ТПО на геомагнитные возмущения не противоречит существующим знаниям.

Долгое время предполагалось, что энергия солнечных заряженных частиц, приходящая к Земле, не может повлиять на состояние нижней атмосферы, так как вся она задерживается магнитосферой. Но не учитывалось, что возникающие при этом возмущения магнитного поля Земли беспрепятственно передают свою энергию электрическим токам в океане. Предстоит еще изучить, в какой мере эти токи влияют на нагрев океана и на отклонение течений от их обычных траекторий.

\section{ЗАКЛЮЧЕНИЕ}

Обнаружены тесные корреляции долгопериодных изменений температуры поверхности Мирового океана в районах основных океанических течений с изменениями планетарной геомагнитной активности. Изменения ТПО в разных частях Мирового океана следовали за изменениями индекса $a a$ с запаздыванием на 22-26 или на 32-33 или на 42-45 лет. Наилучшие связи обнаружились в местах пересечения океаническими течениями препятствий в виде подводных хребтов или гряд ост- 
ровов. Участие в формировании описанных связей подводных препятствий на больших глубинах, усилили предположение об участии глубинных слоев океана в формировании механизма передачи энергии, сопутствующей планетарным геомагнитным возмущениям, в изменения ТПО Мирового океана. Многолетнее запаздывание реакции ТПО на изменения $а a$ предположительно объясняется временем передачи возмущений в океане с больших глубин на поверхность.

\section{СПИСОК ЛИТЕРАТУРЫ}

1. Бялко А.В. Релаксационная теория климата // Успехи физических наук, 2012, т. 182, № 1, с. 111-116.

2. Лаппо С. С. К вопросу о причинах адвекции тепла на север через экватор в Атлантическом океане // Исследование процессов взаимодействия океана и атмосферы. М., 1984, с. 125-129.

3. Лаппо С. С. Добролюбов С. А., Лозовацкий И. Д., Морозов Е. Г., Соков А. В., Шаповалов С. М. Трансформация вод антарктического происхождения и меридиональный перенос в Атлантике к северу от экватора по данным квази-зонального разреза 2000 г. // Фундаментальные исследования океанов и морей. Т. 1, М., Наука, 2006, с. 15-32.

4. Пилипенко В.А. Белаховский В.Б., Селиванов В.Н., Сахаров Я.А. Характеристики вариабельности геомагнитного поля для изучения воздействия магнитных бурь и суббурь на электроэнергетические системы // Физика Земли, 2018, № 1, с. 56-68.

5. Сушко В. А., Косых Д. А. Геомагнитные штормы. Угроза национальной безопасности России // Новости Электротехники, 2013, № 4 (82), с. 25-28.

6. Шерстюков Б. Г., Переведенцев Ю.П. Дальние асинхронные связи в долгопериодных колебаниях климата Мирового океана и региональной атмосферы применительно к Республике Татарстан // Вестник Воронежского государственного университета. Серия География. Геоэкология, 2019, № 1, с. 5-11.

7. Broecker W.S. The great ocean conveyor // Oceanography, 1991, no. 4(2), pp. 79-89.

8. Climate Change 2013. The Physical Science Basis. Working Group I Contribution to the Fifth Assessment Report of the Intergovernmental Panel on Climate Change. Edited by Thomas F. Stocker, Dahe Qin, Gian-Kasper Plattner, Melinda M.B. Tignor, Simon K. Allen, Judith Boschung, Alexander Nauels, Yu Xia, Vincent Bex, Pauline M. Midgley. Cambridge, New York, Melbourne, Madrid, Cape Town, Singapore, Sго Paolo, Delhi, Mexico City. 1535p. URL: http://www.ipcc.ch/pdf/assessment-report/ar5/ wg1/WG1AR5_ALL_FINAL.pdf

9. Kelly G. S., Viljanen A., Beggan C., Thomson A.W.P. Un-derstanding GIC in the UK and French high voltage transmis-sion systems during severe magnetic storms. Space Weather, 2017, v. 14, iss. 1, pp. 99-114. DOI: 10.1002/ 2016SW001469
10. Mayaud Pierre-Noel. The aa Indices: A 100-Year Series Characterizing the Magnetic Activity // Journal of Geophysical Research, 1972, v. 77, no. 34, pp. 6870-6874, URL: http://isgi.unistra.fr/indices_dst.php

11. Pirjola R., Pulkkinen A., Viljanen A. Studies of space weather effects on the finnish natural gas pipeline and on the finnish high-voltage power system // Advansed Space Research, 2003, vol. 31, issue 4, pp. 795-805. DOI: 10.1016/ S0273-1177(02)00781-0

12. SST data: NOAA Extended Reconstruction SSTs, version 3 (ERSSTv3 \& 3b). Retrieved from https:// climatedataguide.ucar.edu/climate-data/sst-data-noaaextended-reconstruction-ssts-version-3-ersstv3-3b.

13. Smith T.M., Reynolds R.W., Peterson T.C., Lawrimore J. Improvements NOAAs Historical Merged Land-Ocean Temp Analysis (1880-2006) // Journal of Climate, 2008, vol. 21, pp. 2283-2296.

14. Swingedouw D. Terray L., Cassou C., Voldoire A., Salas-Melia D., Servonnat J. Natural forcing of climate during the last millenium: Fingerprint of solar variability. Low frequency solar forcing and NAO // Climate Dynamics, Springer, 2011, vol. 36, pp. 1349-1364.

\section{REFERENCES}

1. Byalko A. V. Relaksatsionnaya teoriya klimata [Relaxation theory of climate]. Uspekhi Fizicheskikh Nauk, 2012, vol. 182, no. 1, pp. 111-116. (In Russ.)

2. Lappo S.S. K voprosu o prichinakh advektsii tepla na sever cherez ekvator $v$ Atlanticheskom okeane [On the causes of advection of heat to the north through the equator in the Atlantic Ocean]. Issledovaniye protsessov vzaimodeystviya okeana i atmosfery. Moscow, 1984, pp. 125-129. (In Russ.)

3. Lappo S. S. Dobrolyubov S. A., Lozovatsky I.D., Morozov E. G., Sokov A. V., Shapovalov S. M. Transformatsiya vod antarkticheskogo proiskhozhdeniya $i$ meridional'nyy perenos $v$ Atlantike $k$ severu ot ekvatora po dannym kvazi-zonal'nogo razreza $2000 \mathrm{~g}$. [Water transformation of Antarctic origin and meridional transport in the Atlantic north of the equator according to the quasi-zonal section of 2000]. In the book Fundamental'nyye issledovaniya okeanov i morey. Moscow, Nauka Publ., 2006, v. 1, pp.15-32. (In Russ.)

4. Pilipenko V. A., Belakhovsky V. B., Selivanov V. N., Sakharov Ya. A. Kharakteristiki variabel'nosti geomagnitnogo polya dlya izucheniya vozdeystviya magnitnykh bur' i subbur' na elektroenergeticheskiye sistemy [Variability characteristics of the geomagnetic field for studying the effects of magnetic storms and substorms on electric power systems]. Fizika Zemli, 2018, no. 1, pp. 56-68. (In Russ.)

5. Sushko V. A., Kosykh D. A. Geomagnitnyye shtormy. Ugroza natsional'noy bezopasnosti Rossii [Geomagnetic storms. Threat to the national security of Russia]. Novosti Elektrotekhniki, 2013, no. 4 (82), pp. 25-28. (In Russ.)

6. Sherstyukov B.G. Perevedentsev Yu.P. Dal'niye asinkhronnyye svyazi v dolgoperiodnykh kolebaniyakh klimata Mirovogo okeana i regional'noy atmosfery 
primenitel'no k Respublike Tatarstan [Long-Range Asynchronous Communications in Long-Period Oscillations of the Climate of the World Ocean and the Regional Atmosphere as Applied to the Republic of Tatarstan]. Vestnik Voronegskogo gosudarstvennogo universiteta. Seriya Geografiya, Geoekologiya, 2019, no. 1, pp. 5-11. (In Russ.)

7. Broecker W. S. The great ocean conveyor. Oceanography, 1991, no. 4(2), pp. 79-89.

8. Climate Change 2013. The Physical Science Basis. Working Group I Contribution to the Fifth Assessment Report of the Intergovernmental Panel on Climate Change. Edited by Thomas F. Stocker, Dahe Qin, Gian-Kasper Plattner, Melinda M.B. Tignor, Simon K. Allen, Judith Boschung, Alexander Nauels, Yu Xia, Vincent Bex, Pauline M. Midgley. Cambridge, New York, Melbourne, Madrid, Cape Town, Singapore, Sго Paolo, Delhi, Mexico City, 1535p, URL: http://www.ipcc.ch/pdf/assessment-report/ar5/wg1/ WG1AR5_ALL_FINAL.pdf

9. Kelly G.S., Viljanen A., Beggan C., Thomson A.W.P. Un-derstanding GIC in the UK and French high voltage transmission systems during severe magnetic storms. Space Weather, 2017, v. 14, iss. 1, pp. 99-114. DOI: 10.1002/ 2016SW001469

\section{КОНФЛИКТ ИНТЕРЕСОВ}

Авторы декларируют отсутствие явных и потенциальных конфликтов интересов, связанных с публикацией настоящей статьи.

Шерстюков Борис Георгиевич

доктор географических наук, старший научный сотрудник Всероссийский НИИ гидрометеорологической информации - Мировой центр данных, г. Обнинск, Калужская обл., Российская Федерация, ORCID: https:// orcid.org/0000-0003-0167-1108, e-mail: boris@ meteo.ru Переведенцев Юрий Петрович

доктор географических наук, профессор Казанского Федерального университета, Института экологии и природопользования, г. Казань, Российская Федерация, ORCID: https://orcid.org/0000-0002-3181-6227
10. Mayaud Pierre-Noel. The aa Indices: A 100-Year Series Characterizing the Magnetic Activity. Journal of Geophysical Research, 1972, vol. 77, no. 34, pp. 68706874, URL: http://isgi.unistra.fr/indices_dst.php

11. Pirjola R., Pulkkinen A., Viljanen A. Studies of space weather effects on the finnish natural gas pipeline and on the finnish high-voltage power system. Advansed Space Research, 2003, vol. 31, iss. 4, pp. 795-805. DOI: 10.1016/ S0273-1177(02)00781-0

12. SST data: NOAA Extended Reconstruction SSTs, version 3 (ERSSTv3 \& 3b). Retrieved from https:// climatedataguide.ucar.edu/climate-data/sst-data-noaa-extended-reconstruction-ssts-version-3-ersstv3-3b

13. Smith T. M., Reynolds R.W., Peterson T. C., Lawrimore J. Improvements NOAAs Historical Merged LandOcean Temp Analysis (1880-2006). Journal of Climate, 2008, no. 21, pp. 2283-2296.

14. Swingedouw D., Terray L., Cassou C., Voldoire A., Salas-Melia D., Servonnat J. Natural forcing of climate during the last millenium: Fingerprint of solar variability. Low frequency solar forcing and NAO. Climate Dynamics, Iss. Springer, 2011, vol. 36, pp. 1349-1364.

\section{CONFLICT OF INTEREST}

The authors declare no information of obvious and potential conflicts of interest related to the publication of this article.

Boris G. Sherstyukov

Dr. Sci. (Geogr.), Researcher at the All-Russian Research Institute of Hydrometeorological Information - World Data Center, Obninsk, Kaluga region, Russian Federation, ORCID: https://orcid.org/0000-0003-0167-1108, e-mail: boris@meteo.ru

Yuryi P. Perevedentsev

Dr. Sci. (Geogr.), Full Prof. of the Kazan Federal University, Institute of Ecology and Nature Management. Kazan, Russian Federation, ORCID: https://orcid.org/0000-0002-3181-6227 